\title{
Film-Coated Tablet plus Tablet Dosage Form
}

National Cancer Institute

\section{Source}

National Cancer Institute. Film-Coated Tablet plus Tablet Dosage Form. NCI Thesaurus.

Code C149515.

Combination package consisting of a film-coated tablet and a tablet. 